\title{
LA INELUDIBLE MONITORIZACIÓN PÚBLICA Y CIVIL DE LA TRANSPARENCIA INFORMATIVA EN ESPAÑA
}

The inescapable public and civic monitoring
of informative transparency in Spain

\author{
MARÍA DÍEZ-GARRIDO \\ Universidad Complutense de Madrid \\ mdiez17@ucm.es
}

Cómo citar/Citation

Díez-Garrido, M. ${ }^{a}(2021)$.

La ineludible monitorización pública y civil de la transparencia en España.

Revista de Estudios Políticos, 194, 189-211

doi: https://doi.org/10.18042/cepc/rep.194.07

\section{Resumen}

Espańa cuenta con una escasa cultura de la transparencia, por lo que la «Ley 19/2013 de transparencia, acceso a la información pública y buen gobierno» tardó en llegar más que en otros países. Desde entonces, ha habido un esfuerzo por parte de las instituciones públicas por abrir portales de datos accesibles. En este contexto, la evaluación de la transparencia se vuelve necesaria para asegurar que se cumple el derecho de los ciudadanos a acceder a la información pública y para asegurar que esta sea de calidad. En Espańa, existen modelos muy diferentes de monitorización de la transparencia. Desde el punto de vista público, está el Consejo de Transparencia y Buen Gobierno y los diferentes comisionados de transparencia puestos en marcha en algunas comunidades autónomas. Por otro lado, existen diversos índices de transparencia que evalúan con indicadores la información publicada por las instituciones. En este artículo, estudiaremos la situación actual de la evaluación de la transparencia en Espańa, analizaremos los fallos y destacaremos mejoras necesarias en este sistema.

\section{Palabras clave}

Transparencia; datos abiertos; fiscalización; monitorización; Consejo de Transparencia y Buen Gobierno. 


\section{Abstract}

Spain has a scarce culture of transparency, so the Law 19/2013 of transparency, access to public information and good government took longer to arrive than in other countries. Since then, there has been an effort by public institutions to launch open data portals that are accessible. In this context, the evaluation of transparency becomes necessary to ensure that the right of citizens to access public information is fulfilled and to guarantee that the information is of good quality. In Spain, there are very different models for monitoring transparency. From the public point of view, there is the Council for Transparency and Good Governance and several transparency commissioners set up in some autonomous communities. On the other hand, there are various transparency indexes that evaluate with indicators the information published by the institutions. In this article, we will study the current situation of the evaluation of transparency in Spain, analyze the failures and highlight necessary improvements to this system.

\section{Keywords}

Transparency; open data; auditing; monitoring; Council of Transparency and Good Government. 


\section{SUMARIO}

I. INTRODUCCIÓN Y OBJETIVOS. II. MARCO TEÓRICO: 1. La cultura de la transparencia en España y sus efectos. 2. La necesidad de evaluar la transparencia. III. LA MONITORIZACIÓN PÚBLICA. IV. EVALUACIONES DE ORGANISMOS CIVILES Y PROPUESTA. V. CONCLUSIONES. Biblografía. Apéndice legislativo.

\section{INTRODUCCIÓN Y OBJETIVOS}

La transparencia es un bien indispensable para una buena gobernanza, que es vista como un elemento que equilibra el poder entre los políticos y la ciudadanía (Matheus et al., 2020). Esta consiste en la provisión de datos al público por parte de las instituciones políticas, de modo que la población esté informada para tomar decisiones y se aporten datos a agentes que lo requieran, como los medios de comunicación (Bertot et al, 2010).

El desarrollo de políticas de transparencia se debe a que esta potencia la rendición de cuentas, que implica la responsabilización por parte de los políticos de sus decisiones y el cumplimiento de sus compromisos con la ciudadanía. Para que la rendición de cuentas sea una realidad, es preciso que los agentes políticos ejecuten su cometido. Esto hace necesaria una monitorización de sus actividades, que permita estudiar y analizar la evolución de la transparencia de las instituciones públicas. Así, la evaluación es imprescindible para el cumplimiento de las políticas de transparencia y para que los políticos rindan cuentas sobre esta cuestión a la población.

El objetivo de este artículo es conocer el sistema de evaluación público y por parte de organismos civiles de la transparencia en España. Se quiere responder a dos preguntas de investigación:

- ¿Qué mejoras necesita el Consejo de Transparencia y Buen Gobierno y los organismos homónimos de las comunidades autónomas?

- ¿Qué metodologías deben usar los organismos civiles para monitorizar la transparencia en España en profundidad?

El artículo sigue la siguiente estructura: en primer lugar, se constituirá un marco teórico que estudie el desarrollo de la transparencia en Espańa y sus posibles consecuencias, así como las razones por las que es necesario evaluar la apertura informativa. A continuación, se analizarán los cauces públicos para 
esta evaluación de la transparencia y, posteriormente, desde el punto de vista civil, de forma que se puedan comparar metodologías y averiguar posibles mejoras en estos sistemas. Finalmente, las conclusiones tratarán de responder a las preguntas de investigación y proponer un camino para la monitorización de la transparencia.

\section{MARCO TEÓRICO}

\section{LA CULTURA DE LA TRANSPARENCIA EN ESPAÑA Y SUS EFECTOS}

La transparencia es una pieza importante en el discurso de los políticos españoles desde hace años y esta se ha hecho presente en sus programas de medidas, así como en los debates políticos. Esta relevancia ha sido aún más evidente con la llegada de la pandemia por la COVID-19, que ha generado reflexiones sobre la necesidad de abrir los datos públicos tanto en el escenario político, como en el mediático y también en el académico.

La cultura de la transparencia en España es escasa y breve, pues su verdadero impulso llegó con la tardía aprobación de la Ley 19/2013 de transparencia, acceso a la información pública y buen gobierno. Delgado Morales et al. (2015) explican que previamente existía un parco interés por la transparencia en España, pero este hecho cambió especialmente con la llegada de la crisis económica en 2007. A esto se sumó, además, el auge tanto en el ámbito político como en el académico de las reflexiones sobre el gobierno abierto, especialmente tras el Memorando por la transparencia y el gobierno abierto del expresidente estadounidense Barack Obama en 2009 (Ruvalcaba-Gómez et al., 2019), que prometió que su administración sería abierta y rendiría cuentas a la ciudadanía. Asimismo, los numerosos casos de corrupción destapados en la última década crearon una conciencia social favorable a la transparencia.

Comenzó así una nueva etapa en la cultura de la transparencia española, que se reflejó con numerosas publicaciones académicas, así como con la aprobación de leyes y la publicación de portales de datos abiertos en casi todas las comunidades autónomas del país (Curto-Rodríguez, 2020).

Todas estas medidas fueron impulsadas también por la Open Government Partnership, una organización internacional que se encarga de promulgar las medidas de gobierno abierto por todo el mundo, y de la que Espańa es miembro desde 2011. En este sentido, la transparencia es uno de los pilares del gobierno abierto, junto a la participación y la colaboración (Ruvalcaba-Gómez et al, 2019), lo que ha conducido al Gobierno de España a no solo impulsar la primera, sino crear también un Plan de Acción de Gobierno Abierto, en el que 
se desarrollen los aspectos que tienen que ver con la interacción de la ciudadanía, la deliberación y la cocreación de políticas. Estos planes han ido avanzando desde que se inició el primero en 2012. Actualmente, se está desarrollando el IV Plan de Acción de Gobierno Abierto, que durará hasta el año 2024. Este plantea medidas como la sensibilización de la población, la formación de los empleados públicos y la mejora del sistema evaluador de la transparencia, que es precisamente el tema que ocupa este artículo.

La transparencia consiste en la publicación de información, de forma que esta sea accesible para la ciudadanía y que las decisiones de los gobernantes puedan ser observadas por los gobernados o votantes (Hood y Heald, 2006). Se debe distinguir, como subrayan Mabillard y Zumofen (2017), entre la transparencia activa, que consiste en la publicación voluntaria de datos de forma estructurada en un portal digital, y pasiva, que permite a la ciudadanía acceder a los datos a través de solicitudes de información y que se regula de acuerdo a unos periodos temporales de respuesta y unos límites temáticos. Así lo reconoce, de hecho, la Ley 19/2013, que no solo estableció una serie de obligaciones de publicidad activa, sino que creó un sistema de peticiones de información.

España y otros países se interesaron en promocionar la transparencia y en aprobar diversas leyes de acceso a la información y en lanzar portales de datos abiertos tras el discurso de Obama (Matheus et al., 2020), puesto que la apertura informativa genera cambios políticos relevantes y siempre se han destacado sus consecuencias positivas (Worthy, 2010; Ruijer et al, 2020).

Destaca entre esos efectos positivos la recuperación de la confianza por parte de la ciudadanía, que en España ha caído en picado en los últimos años, como destacan periódicamente el CIS y el Eurobarómetro. Para Cahlikova y Mabillard (2020), la transparencia estrecha los lazos entre el poder y la población, a través del intercambio de información entre las dos partes. Una ciudadanía más informada tomará decisiones mejores en cuanto a sus representantes políticos, de modo que se desarrollará la rendición de cuentas y se incrementará la mencionada confianza en las instituciones públicas, como señalan estas autoras.

La transparencia y la recuperación de la confianza pueden producir una mejor gobernanza y esta, a su vez, deriva en el crecimiento económico de las democracias que la desarrollan (Janssen et al., 2012). Esto se debe a que la apertura de datos implica una mayor eficacia de las instituciones y, por tanto, impulsa el ahorro de costes y una aplicación más efectiva de los gastos públicos.

Matheus et al. (2020) también señalan la lucha contra la corrupción como una de las ventajas de la apertura informativa. Berliner (2014) afirma que, cuando los políticos son los propietarios de la información, es más sencillo cometer fraudes. Sin embargo, cuando la información pasa a ser pública y es conocida por la ciudadanía, se vuelve más difícil. La transparencia, por tanto, no es una antídoto infalible contra la corrupción, pero sí la 
dificulta y ayuda a concienciar a la población y a la clase política sobre la necesidad de ser claro y honesto en la ejecución de las políticas públicas.

La apertura informativa encuentra uno de sus principales apoyos en la tecnología, que evoluciona a pasos agigantados. Las instituciones no deben perder de vista la inteligencia artificial y sus modelos pueden beneficiarse del big data, de modo que esta smartificación de las administraciones conlleve una renovación de las estructuras políticas, como sugiere Ramió (2019). Por tanto, la transparencia también puede tener un efecto positivo en la regeneración democrática y, de hecho, esta es una de las justificaciones que aporta la Ley 19/2013 en su preámbulo:

Los países con mayores niveles en materia de transparencia y normas de buen gobierno cuentan con instituciones más fuertes, que favorecen el crecimiento económico y el desarrollo social. En estos países, los ciudadanos pueden juzgar mejor y con más criterio la capacidad de sus responsables públicos y decidir en consecuencia. Permitiendo una mejor fiscalización de la actividad pública se contribuye a la necesaria regeneración democrática, se promueve la eficiencia y eficacia del Estado y se favorece el crecimiento económico ${ }^{1}$.

La transparencia, además, es una de las soluciones más eficaces ante la desinformación, un fenómeno que los Gobiernos y los ciudadanos no pueden ni deben olvidar. Esta no solo se limita a la propagación de noticias falsas aisladas, como afirman Bennet y Livingston (2018), sino que la polarización mediática y política han provocado un contexto social en el que los argumentos se basan en las emociones y la manipulación. La transparencia y su regulación suponen en este sentido un acceso a información veraz que es necesaria, según Magallón Rosa (2019), para ejercer prácticas contra la desinformación, como el periodismo de verificación o fact-checking.

Recuperación de la confianza, eficiencia de las políticas, crecimiento económico, disminución de la corrupción, regeneración democrática y lucha contra la desinformación. Estos son algunos de los efectos positivos que los autores destacan de la transparencia y que hacen necesario estudiar y evaluar su desarrollo.

\section{LA NECESIDAD DE EVALUAR LA TRANSPARENCIA}

Como cualquier actividad pública, la transparencia es susceptible de ser estudiada y controlada para asegurar su cumplimiento. Las instituciones pueden

1 Ley 19/2013 de transparencia, acceso a la información pública y buen gobierno. 
aparentar ser transparentes para observadores externos, pero mantener prácticas opacas (Ruijer et al, 2020), a través de datasets incomprensibles y otras medidas similares. Por ello, es preciso ejercer presión sobre las Administraciones públicas para que sean transparentes y sus portales de datos mantengan una calidad considerable, a través de evaluaciones que no caigan en falsas apariencias y analicen en profundidad la apertura informativa.

La evaluación de la transparencia que se plantea en este artículo se basa en los principios de la democracia monitorizada. En esta nueva era marcada por la tecnología, como indica Keane (2009), los ciudadanos no solo pueden controlar al poder a través del sistema de representación tradicional, sino que se pueden volver watchdogs del poder, auditar, hacer un escrutinio y observar a través de múltiples canales las decisiones tomadas por los poderes públicos. Feenstra (2012) afirma que para que la democracia sea posible, esta tiene que estar controlada, debe ser evaluada por los ciudadanos y esta monitorización presionará a los políticos a ejercer su poder de manera correcta.

Los millones de flujos informativos posibles gracias a internet incrementan las opciones de monitorizar al poder en la línea de lo que seńalan estos autores. Aplicando este concepto a la transparencia informativa, su evaluación se vuelve indispensable para su propia evolución y, dado que la transparencia se desarrolla a través de internet, su control puede efectuarse también a través de herramientas y canales digitales.

Esto no solo permite a los ciudadanos realizar un escrutinio de las decisiones públicas, sino que también beneficia a las instituciones. Pernagallo y Torrisi (2020) afirman que, igual que las empresas realizan auditorías externas para conocer los intereses de sus clientes y analizar sus recursos, las Administraciones deben controlar sus mecanismos y estructuras para responder a las necesidades de la ciudadanía. Esto ayudará a que la transparencia se ejecute adecuadamente $y$, por tanto, tenga las consecuencias anteriormente mencionadas, como la regeneración democrática, la recuperación de la confianza y la lucha contra la corrupción. Ya en 2010, Worthy mencionaba que las leyes de acceso a la información casi siempre disponían de algún sistema de control: «En el centro de cada ley está el derecho a solicitar el acceso a la información gubernamental, sujeto a ciertas restricciones, y a menudo, un sistema de apelación externa en forma de comisionado, tribunal o el sistema de cortes» (Worthy, 2010: 561).

Existe un claro interés por evaluar y medir la transparencia desde muy diferentes puntos de vista, entre los que destacan el ámbito público, académico y el civil. En este sentido, se han creado diversos organismos públicos que evalúan la transparencia de las instituciones políticas españolas, especialmente desde la aprobación de la Ley 19/2013. Esta supuso la creación del Consejo de Transparencia y Buen Gobierno, que se analizará en el próximo 
epígrafe, cuya finalidad es precisamente asegurar el correcto cumplimiento de la normativa.

Al tiempo que se aprobó la Ley 19/2013, diversas comunidades fueron aprobando sus respectivas leyes de carácter autonómico y algunas implicaron también la creación de consejos y comisionados de transparencia, con funciones similares a la estatal, pero adaptados a su ámbito territorial.

Frente a esta evaluación pública de la transparencia de las instituciones políticas españolas, este fenómeno también ha despertado el interés de numerosos estudios académicos, así como de organismos civiles que fiscalizan la actividad de los políticos. Son organismos como la conocidísima ONG Transparencia Internacional, que disponen de sus propios rankings e índices de transparencia.

Varios estudios ya han analizado estas mediciones, como los de Sierra (2018) y Ros Medina (2018), que concluyen que existe una clara ausencia de consenso metodológico entre ellos y que se trata de un sistema que prima la cantidad de información publicada por las entidades, respecto a la calidad de la misma. Molina et al. (2017) indican que el modelo es predominantemente cuantitativo y subrayan la necesidad de dar peso a las metodologías que evalúen la transparencia: «El procedimiento ha de ser capar de definir con precisión las características con que se ha de ofrecer la información relativa a cada indicador para ser validado. Estas características no son solo técnicas y formales, sino que han de considerar otras cualidades de la transparencia como la inteligibilidad de las informaciones y la facilidad de acceso» (Molina et al., 2017: 828).

Ros Medina (2018) destaca, además, la relevancia que adquieren estos análisis en los medios de comunicación, que provoca que las instituciones con mejores resultados en estos índices se vean reforzados públicamente gracias a la difusión de dichos estudios.

Esta presencia de los rankings de transparencia en los medios de comunicación y redes sociales, según destacan Delgado Morales et al. (2015), redunda en que las webs de las instituciones se basen en las estructuras de estos índices, de manera que puedan obtener un buen resultado y así aparecer en los titulares informativos como «los más transparentes».

\section{LA MONITORIZACIÓN PÚBLICA}

Para rendir cuentas a la ciudadanía, se debe controlar que la transparencia se cumple y, por este motivo, existen organismos de carácter público que se dedican a ejercer esa monitorización de la apertura informativa. Como se ha mencionado anteriormente, el Consejo de Transparencia y Buen 
Gobierno se creó tras la aprobación de la Ley 19/2013 y, además, existen órganos equivalentes en las comunidades autónomas. En este apartado, se pretenden explorar las siguientes cuestiones sobre los organismos públicos de control de la transparencia:

- Adecuación de las metodologías utilizadas.

- Control de la transparencia más allá del cumplimiento de las normas.

- Aciertos y fallos del sistema.

- Posibles mejoras.

Sin duda, el organismo público de mayor relevancia en este apartado es el CTBG, cuyo funcionamiento se regula en el Real Decreto 919/2014, de 31 de octubre, por el que se aprueba el Estatuto del Consejo de Transparencia y Buen Gobierno. Según este estatuto, sus funciones principales son: evaluar el cumplimiento de las normas relativas a la transparencia, elaborar informes y memorias sobre buenas prácticas, asesorar en materia de transparencia, emitir recomendaciones sobre la aplicación de las normas de apertura informativa, orientar acerca de los proyectos normativos de transparencia del país, formar y sensibilizar en estas materias y colaborar con órganos de naturaleza similar.

Imagen 1. Funciones del Consejo de Transparencia y Buen Gobierno

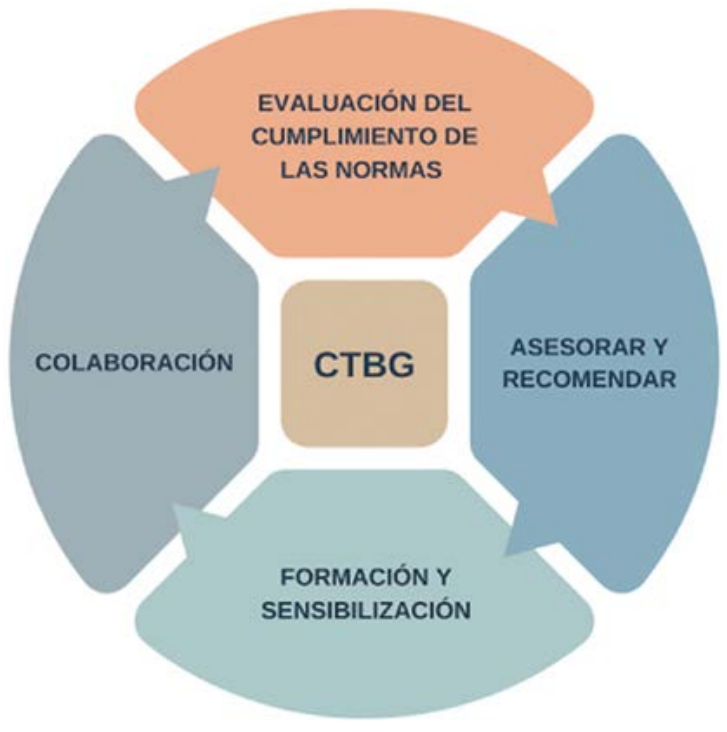

Fuente: elaboración propia. 
Uno de los fallos principales en la concepción del CTBG es que en el propio RD 919/2014 se asume su funcionamiento como un gasto, en lugar de una inversión. La disposición adicional primera expresa que «la creación y puesta en funcionamiento del Consejo de Transparencia y Buen Gobierno no podrán suponer incremento de dotaciones, retribuciones, $\mathrm{u}$ otros gastos de personal ni, por otros conceptos, incremento neto de estructura o de personal al servicio del sector público estatal». Ello limita enormemente las capacidades del personal del CTBG, que se ve superado por sus numerosas funciones y no encuentra posibilidad alguna de crecimiento.

El RD 919/2014 establece el régimen jurídico del Consejo, su estructura orgánica, su régimen económico y patrimonial y la prestación de servicios que pueda requerir por parte de la de la Abogacía General del Estado-Dirección del Servicio Jurídico del Estado. Una de las cuestiones fundamentales es el nombramiento de la persona que ocupe la presidencia del CTBG, que debe ser propuesta por el titular del Ministerio de Hacienda y Administraciones Públicas, debe comparecer ante la comisión competente del Congreso de los Diputados y ser refrendado en las Cámara Baja por mayoría absoluta. El Estatuto indica que la persona debe contar con un reconocido prestigio y ocupará el cargo por un periodo de cinco años que no es renovable.

En esta línea, cabe destacar lo ignorado que ha sido el CTBG durante años, pues tras el fallecimiento de la primera presidenta, Esther Aritzmendi, en 2017, Francisco Javier Amorós se encargó de las funciones de presidente, que previamente ocupaba el cargo de subdirector y cobró retribuciones como $\mathrm{tal}^{2}$ aunque ejerciera de presidente en funciones. Este puesto provisional se alargó casi tres años, hasta que José Luis Rodríguez Álvarez se convirtió en el nuevo presidente a finales del año 2020. Esto demuestra la poca importancia que otorgan los políticos a este organismo, así como el escaso consenso político al respecto, pues durante un largo periodo de tiempo no han acordado el nombramiento de una persona, a pesar de que el propio CTBG lo reclamaba en la Memoria Anual de 2018.

El CTBG desarrolla sus funciones fiscalizadoras de la transparencia a través de la conocida Metodología de evaluación y seguimiento de la transparencia de la actividad pública, más conocida como MESTA, diseñada en colaboración con la Agencia Estatal de Evaluación de las Políticas Públicas y la Calidad de los Servicios. Esta metodología pretende establecer unos criterios para analizar tanto la publicidad activa como el derecho de acceso a la información, que sea aplicable a todos los sujetos obligados. Ello supone un reto

2 Disponible en: https:/www.newtral.es/consejo-transparencia-buen-gobierno-presidente/ 20201001/. 
tremendamente complejo, pues, además de la Ley 19/2013, la mayoría de comunidades autónomas cuentan con una regulación propia y diferente de la transparencia. Este grado de dificultad es reconocido en la memoria de la metodología MESTA, donde recalcan la irregular aplicación de leyes autonómicas y la diversidad de los sujetos obligados a cumplirlas.

MESTA estudia la publicidad activa conforme a tres criterios: la publicación de la información (contenido, forma y actualización), los atributos de la misma (accesibilidad, claridad, estructura, reutilización) y las características del soporte o canal en el que se difunde. Además, se establece un mapa de publicidad activa, según las obligaciones aplicables a cada sujeto. Por ejemplo, no debe evaluarse igual al Ayuntamiento de Manzanal de Arriba (Zamora), que cuenta con menos de quinientos habitantes, que el Ministerio de Educación. Por este motivo, la metodología incluye un cuestionario de actividades, para que cada entidad pueda identificar la información que debe publicar. También cuenta con un apartado con el resto de criterios ya mencionados, como el soporte web.

Esto implica la colaboración activa de las instituciones, lo que supone un gran reto, como destaca Ros Medina (2018), pues los sujetos obligados más grandes cuentan con incentivos para responder al cuestionario con los que no cuenta uno de menor relevancia. El mismo autor destaca que MESTA no establece ninguna solución si una institución no responde al cuestionario o si falsea las obligaciones que tiene que cumplir.

El análisis que propone MESTA contiene dos indicadores: el indicador de cumplimiento en publicidad activa (ICPA), que clasifica el grado de cumplimiento de las obligaciones de publicidad activa en las normas; y el indicador de transparencia en publicidad activa (ITPA), que valora el nivel de transparencia conforme a la información que no es obligatoria.

La aplicación de esta metodología se puede observar en las memorias anuales del CTBG. Sin embargo, la Memoria de $2019^{3}$ señala algunos límites, como la amplia cantidad de sujetos que deben cumplir la Ley 19/2013, que les obligó a evaluar muestras estadísticas y, por tanto, solo permite esbozar la situación general de la transparencia. Así, el CTBG, que tiene a su cargo funciones muy importantes respecto a la fiscalización de la transparencia, no cuenta con incentivos ni capacidad suficiente para realizar lo que se le pide y, además, el reglamento dificulta enormemente incrementar estas opciones, debido a la limitación mencionada anteriormente.

En esta memoria, el CTBG explica la intensa evaluación realizada a algunos organismos con detalles interesantes, donde destacan cuestiones como

3 Consejo de Transparencia y Buen Gobierno (2020). 
los desfases temporales de la información publicada por algunas instituciones o el compromiso de otras por aportar información adicional a la que exige la ley.

Las memorias también incluyen un resumen de otra de las funciones del CTBG, que es atender a las reclamaciones de los ciudadanos respecto al derecho de acceso a la información pública. El organismo indica el crecimiento cuantitativo del número de reclamaciones en esta materia, donde señalan también el descenso en la tasa de conflictividad de las mismas. Sin embargo, el propio CTBG reconoce que no se puede llegar a conclusiones válidas sin comparar estos datos en un examen paralelo en el ámbito autonómico, de modo que resulta imprescindible la colaboración entre ellos.

Llama la atención, aun así, que las memorias del propio CTBG incumplen el principio de accesibilidad, pues solo son descargables en formato PDF. En este sentido, las memorias explican los resultados obtenidos por algunas instituciones y organismos, pero sería muy interesante garantizar el acceso a los resultados también en formatos reutilizables. Ello permitiría a agentes externos usar estos datos y monitorizar el cumplimiento de la normativa. Puede incluso beneficiar a las instituciones para mejorar sus resultados y comprender el funcionamiento de estas evaluaciones.

MESTA resulta una interesante metodología para los organismos de ámbito. Sin embargo, cuenta con algunas cuestiones mejorables. La más relevante es que, al ser una metodología algo cerrada, que dificulta la evaluación de situaciones no previstas por las instituciones, como puede ser la pandemia por la COVID. En este sentido, sería interesante contar con un método público de monitorización que actualice constantemente las actividades ejemplares en este sentido.

MESTA es una metodología esencialmente cuantitativa y muestra un resultado final en forma de número de cada uno de sus indicadores, con un máximo de 100 puntos. Si bien es importante contar con una medición cuantitativa y, por tanto, objetiva, sería interesante también avanzar en una metodología mixta, que combinara estudios cuantitativos y cualitativos. Así, no solo se tendría que analizar la calidad de la información publicada en los portales de transparencia, sino también otras cuestiones como la forma de trabajar en estos. Ello permitiría avanzar notoriamente en la evaluación de la transparencia y permitir a las instituciones aprender de los aciertos y errores de otras entidades.

Por otro lado, aunque se trata de una metodología muy completa, que no solo tiene en cuenta la información publicada, sino la forma en la que se publica, el CTBG no tiene capacidad para aplicarla. Son tantos los sujetos obligados a cumplir con la Ley 19/2013 y son tantas las limitaciones técnicas y económicas del Consejo, que resulta imposible emplearla como se debe. 
Además, es imprescindible la colaboración con los consejos y comisionados de la transparencia en las autonomías de todo el país. Las comunidades cuentan con leyes propias y, por tanto, con sus propios organismos garantes de ese derecho. En la siguiente tabla se indica la norma y el consejo de cada autonomía:

Tabla 1. Leyes y consejos de transparencia de cada comunidad autónoma

\begin{tabular}{|c|c|c|}
\hline & Ley de transparencia & Consejo o comisionado \\
\hline Andalucía & $\begin{array}{l}\text { Ley } 1 / 2014 \text {, de } 24 \text { de junio, de } \\
\text { Transparencia Pública de Andalucía }\end{array}$ & $\begin{array}{l}\text { Consejo de Transparencia } \\
\text { y Protección de Datos de } \\
\text { Andalucía }\end{array}$ \\
\hline Aragón & $\begin{array}{l}\text { Ley } 8 / 2015 \text {, de } 25 \text { de marzo, de } \\
\text { transparencia de la actividad pública y } \\
\text { participación ciudadana de Aragón }\end{array}$ & $\begin{array}{c}\text { Consejo de Transparencia } \\
\text { de Aragón }\end{array}$ \\
\hline $\begin{array}{l}\text { Principado } \\
\text { de Asturias }\end{array}$ & $\begin{array}{c}\text { Ley } 8 / 2018 \text {, de } 14 \text { de septiembre, de } \\
\text { transparencia, buen gobierno y grupos } \\
\text { de interés }\end{array}$ & $\begin{array}{l}\text { Sin constituirse. Convenio } \\
\text { con el CTBG }\end{array}$ \\
\hline Illes Balears & $\begin{array}{c}\text { Ley } 3 / 2017 \text {, de } 7 \text { de julio, de } \\
\text { modificación de la Ley } 20 / 2006 \text {, } \\
\text { de } 15 \text { de diciembre, municipal y de } \\
\text { régimen local de las Illes Balears, para } \\
\text { introducir medidas de transparencia y } \\
\text { participación }\end{array}$ & $\begin{array}{l}\text { Comisión para las } \\
\text { Reclamaciones de Acceso } \\
\text { a la Información Pública }\end{array}$ \\
\hline Canarias & $\begin{array}{c}\text { Ley } 12 / 2014 \text {, de } 26 \text { de diciembre, } \\
\text { de transparencia y de acceso a la } \\
\text { información pública }\end{array}$ & $\begin{array}{c}\text { Comisionado de } \\
\text { Transparencia y Acceso a } \\
\text { la Información Pública de } \\
\text { Canarias }\end{array}$ \\
\hline Cantabria & $\begin{array}{l}\text { Ley de Cantabria } 1 / 2018 \text {, de } 21 \\
\text { de marzo, de transparencia de la } \\
\text { actividad pública }\end{array}$ & $\begin{array}{l}\text { Sin constituirse. Convenio } \\
\text { con el CTBG }\end{array}$ \\
\hline $\begin{array}{l}\text { Castilla y } \\
\text { León }\end{array}$ & $\begin{array}{c}\text { Ley } 3 / 2015 \text {, de } 4 \text { de marzo, de } \\
\text { transparencia y participación } \\
\text { ciudadana de Castilla y León. } \\
\text { NUEVA LEY EN TRÁMITE } \\
\text { Aprobado el Proyecto de Ley } \\
\text { de Transparencia, acceso a la } \\
\text { información pública y su reutilización } \\
\text { de la Comunidad de Castilla y León } \\
\end{array}$ & $\begin{array}{l}\text { Comisionado de } \\
\text { Transparencia de Castilla } \\
\text { y León }\end{array}$ \\
\hline $\begin{array}{l}\text { Castilla-La } \\
\text { Mancha }\end{array}$ & $\begin{array}{l}\text { Ley } 4 / 2016 \text {, de } 15 \text { de diciembre, de } \\
\text { transparencia y buen gobierno de } \\
\text { Castilla-La Mancha }\end{array}$ & $\begin{array}{l}\text { Sin constituirse. Convenio } \\
\text { con el CTBG }\end{array}$ \\
\hline
\end{tabular}




\begin{tabular}{|c|c|c|}
\hline & Ley de transparencia & Consejo o comisionado \\
\hline Cataluña & $\begin{array}{l}\text { Ley } 19 / 2014, \text { de } 29 \text { de diciembre, } \\
\text { de transparencia, acceso a la } \\
\text { información pública y buen } \\
\text { gobierno de Cataluña }\end{array}$ & $\begin{array}{l}\text { Comissió de Garantia } \\
\text { del Dret d'Accés a la } \\
\text { Informació Pública y } \\
\text { Síndic de Greuges }\end{array}$ \\
\hline $\begin{array}{l}\text { Comunitat } \\
\text { Valenciana }\end{array}$ & $\begin{array}{l}\text { Ley } 2 / 2015 \text {, de } 2 \text { de abril, de } \\
\text { transparencia, buen gobierno y } \\
\text { participación ciudadana de la } \\
\text { Comunitat Valenciana }\end{array}$ & $\begin{array}{l}\text { Consejo de Transparencia, } \\
\text { Acceso a la Información } \\
\text { Pública y Buen Gobierno }\end{array}$ \\
\hline Extremadura & $\begin{array}{l}\text { Ley } 4 / 2013 \text {, de } 21 \text { de mayo, de } \\
\text { Gobierno Abierto de Extremadura }\end{array}$ & $\begin{array}{c}\text { Sin constituirse. Convenio } \\
\text { con el CTBG }\end{array}$ \\
\hline Galicia & $\begin{array}{l}\text { Ley } 1 / 2016 \text {, de } 18 \text { de enero, de } \\
\text { transparencia y buen gobierno }\end{array}$ & $\begin{array}{l}\text { Comisión de } \\
\text { Transparencia de Galicia }\end{array}$ \\
\hline $\begin{array}{l}\text { Comunidad } \\
\text { de Madrid }\end{array}$ & $\begin{array}{l}\text { Ley } 10 / 2019 \text { de } 10 \text { de abril, de } \\
\text { transparencia y de participación de la } \\
\text { Comunidad de Madrid }\end{array}$ & $\begin{array}{c}\text { Consejo de Transparencia } \\
\text { y Participación de la } \\
\text { Comunidad de Madrid }\end{array}$ \\
\hline
\end{tabular}

Ley 7/2016, de 18 de mayo, de

Reforma de la Ley 12/2014, de

Región de 16 de diciembre, de transparencia Murcia y participación ciudadana de la

Comunidad Autónoma de la Región

de Murcia

\begin{tabular}{lcc}
\hline $\begin{array}{l}\text { Comunidad } \\
\text { Foral de } \\
\text { Navarra }\end{array}$ & $\begin{array}{c}\text { Ley Foral 5/2018, de 17 de mayo, de } \\
\text { transparencia, acceso a la información } \\
\text { pública y buen gobierno }\end{array}$ & $\begin{array}{c}\text { Consejo de Transparencia } \\
\text { de Navarra }\end{array}$ \\
\hline País Vasco & En trámite & $\begin{array}{c}\text { Comisión Vasca de Acceso } \\
\text { a la Información Pública }\end{array}$ \\
\hline La Rioja & $\begin{array}{c}\text { Ley 3/2014, de 11 de septiembre, de } \\
\text { transparencia y buen gobierno de La } \\
\text { Rioja }\end{array}$ & $\begin{array}{c}\text { Sin constituirse. Convenio } \\
\text { con el CTBG }\end{array}$ \\
\hline $\begin{array}{l}\text { Ciudad } \\
\text { Autónoma } \\
\text { de Ceuta }\end{array}$ & No existe. & $\begin{array}{c}\text { Sin constituirse. Convenio } \\
\text { con el CTBG }\end{array}$ \\
\hline $\begin{array}{l}\text { Ciudad } \\
\text { Autónoma } \\
\text { de Melilla }\end{array}$ & $\begin{array}{c}\text { No existe, pero hay un Reglamento } \\
\text { info transparencia y acceso a la } \\
\text { Autón pública de la Ciudad }\end{array}$ & $\begin{array}{c}\text { Sin constituirse. Convenio } \\
\text { con el CTBG }\end{array}$ \\
\hline
\end{tabular}

Fuente: elaboración propia. 
Esta larga lista demuestra, en primer lugar, una gran desigualdad entre las comunidades autónomas que cuentan con una ley de transparencia y las que no, así como con las que disponen de un organismo de control. Esto, además, amplía las competencias del Consejo de Transparencia y Buen Gobierno nacional que, a través de convenios con esas autonomías, ejerce de comisionado de la transparencia en dichos territorios. Así ocurre, por ejemplo, con Cantabria.

Precisamente en el V Congreso Internacional de la Transparencia celebrado de forma online en octubre de 2020, la Red de Consejos y Comisionados de Transparencia - compuesta por el CTBG y sus órganos equivalentes en las autonomías - emitió una declaración donde criticaban especialmente aquellas comunidades que aún no cuentan con uno, pero sí disponen de una ley. Según los consejos de transparencia, esto representa «una muestra de incoherencia entre el sistema autonómico de transparencia que se dice y presume configurar por ley autonómica y el realmente "no puesto en marcha", en la práctica» ${ }^{4}$.

En esta declaración, la Red de Consejos y Comisionados de Transparencia de España también manifestó la intención de apoyar una reforma de la Ley 19/2013, desarrollarla y dirigir su camino también hacia las medidas anti-corrupción y las políticas de participación ciudadana. En esta línea, consideramos imprescindible que los políticos cuenten con el asesoramiento y las ideas de estos organismos — sumado al de otros agentes políticos y sociales - para reformar las normas, ya que estos conocen muy bien las exigencias de la ciudadanía y las mejoras que tendrían más efectos beneficiosos en la sociedad.

Sin duda, un asunto pendiente es la monitorización del gobierno abierto, pues también debe ser evaluado. El Portal de Transparencia del Gobierno de Espańa informa sobre los avances en su ejecución. Recientemente, ha despegado el IV Plan de Acción de Gobierno Abierto, de modo que aún no existe una evaluación del mismo, pero sí se puede visitar la fiscalización de los planes anteriores. De esta forma, el Portal de Transparencia rendía cuentas sobre el desarrollo de los principios de gobierno abierto explicando en qué consistía cada punto, la fecha de inicio y la final (o su estimación), así como la fase de ejecución en la que se encontraba:

Cumplen así una labor muy necesaria que se debe seguir cuidando, actualizando de forma constante y que sirve para ejercer presión sobre los actores y que, por tanto, se cumplan los objetivos de cada plan de gobierno abierto. Los consejos y comisionados de las comunidades autónomas podrían ayudar en esta función y ofrecer también datos al respecto de cada territorio.

4 Red de Consejos y Comisionados de Transparencia (2020). Declaración de la UNED. Disponible en: https://cutt.ly/qRbiJ0i. 
Imagen 2. Evaluación del III Plan de Acción de Gobierno Abierto

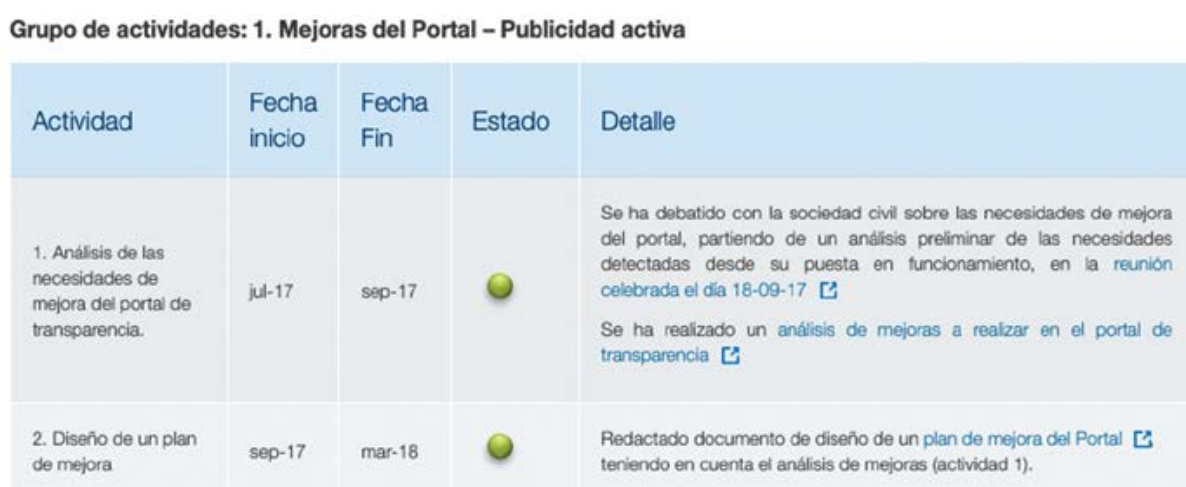

Fuente: Portal de Transparencia del Gobierno de España.

\section{EVALUACIONES DE ORGANISMOS CIVILES Y PROPUESTA}

En paralelo a los consejos y comisionados públicos, existen múltiples organismos de diferente índole que tratan de evaluar el cumplimiento de la transparencia por parte de las instituciones. Existen muchas preguntas en torno a estos y en este apartado se pretende abordar la siguiente información:

— Tipos de organismos civiles que evalúan la transparencia en España

— Adecuación de sus metodologías al marco actual.

- Aciertos y fallos de estos organismos en su forma de trabajar.

- Posibles mejoras de los organismos que monitorizan la transparencia en España.

Antes de la aprobación de la Ley 19/2013, ya comenzaron a surgir diferentes organismos que evalúan la transparencia de las instituciones y diferentes entidades en España, aunque su verdadero auge se dio con la aprobación de la normativa.

Posiblemente la más conocida en este ámbito sea Transparencia Internacional, que está constituida en países de todo el mundo. Se fundó en 1993 y su primera sede se ubicó en Berlín, aunque no tardó en extenderse. Su función fundamental es medir y mitigar los efectos de la corrupción y el fortalecimiento de la integridad política. En esta línea, Transparencia Internacional España dedica parte de sus esfuerzos a evaluar la apertura informativa de las instituciones públicas y cuenta con numerosos índices, como el índice de transparencia de los ayuntamientos (ITA), el índice de transparencia de las 
comunidades autónomas (INCAU), el índice de transparencia de la gestión del agua (INTRAG), el índice de transparencia de las diputaciones (INDIP), y el índice de transparencia de los parlamentos (IPAR), entre otros.

Estos índices cuentan con una cantidad específica de indicadores, que representan un tipo de información que debe ser publicada por las instituciones evaluadas, que se valoran cuantitativamente, Así, cada elemento se cumplimenta con un 0 si la institución no dispone de ello en su página web, un 1 cuando sí cuenta con ello y un 0,5 en caso de que solo lo haga de forma parcial. Según explican en la metodología del ITA, evalúan los indicadores y se lo envían a cada ayuntamiento, para que este pueda incorporar informaciones que desde TI no han incluido (deben enviar el enlace en el que se encuentra dicha información). Finalmente, se suman todos los indicadores obtenidos por cada una de las instituciones monitorizadas y se obtiene una clasificación o ranking comparativo.

De forma similar, la Fundación Haz (antes Compromiso Empresarial y Compromiso y Transparencia), fue compuesta en 2007 por profesionales y académicos cuyo objetivo, según su página web ${ }^{5}$, es reforzar la confianza de la ciudadanía en las instituciones a través de la transparencia y el buen gobierno. Comenzaron realizando un índice de transparencia de las fundaciones españolas en 2009 y, con el paso de los años, incorporaron nuevos organismos a sus informes: medios de comunicación, museos, partidos políticos, universidades, consejos de administración de las empresas del IBEX 35, etc. Realizan también otras guías e informes sobre buen gobierno y apertura informativa.

Los informes de la Fundación Haz clasifican a las instituciones monitorizadas en tres categorías, según el porcentaje de apertura informativa obtenido, que son: transparente, translúcido y opaco. Al igual que TI, valora los indicadores según su tipo cumplimiento por parte de las instituciones: de forma íntegra, parcial y su incumplimiento.

Tanto en el caso de la ONG Transparencia Internacional, como en el de la Fundación Compromiso y Transparencia, resulta muy interesante la repetición de las metodologías a lo largo de los años, que permite comprobar la evolución de los organismos evaluados. De hecho, esta es una de las fortalezas de la Fundación Compromiso y Transparencia, que muestra de forma abierta la evolución de cada organización:

Como se puede ver en el ejemplo gráfico, la Fundación Haz no solo elabora un gráfico, sino que además permite descargar los datos en formato CSV, lo cual supone una muestra notable de transparencia en sí misma.

5 Disponible en: https://www.compromisoytransparencia.com/. 
Imagen 3. Evolución de la apertura informativa de un organismo en la Fundación Compromiso y Transparencia

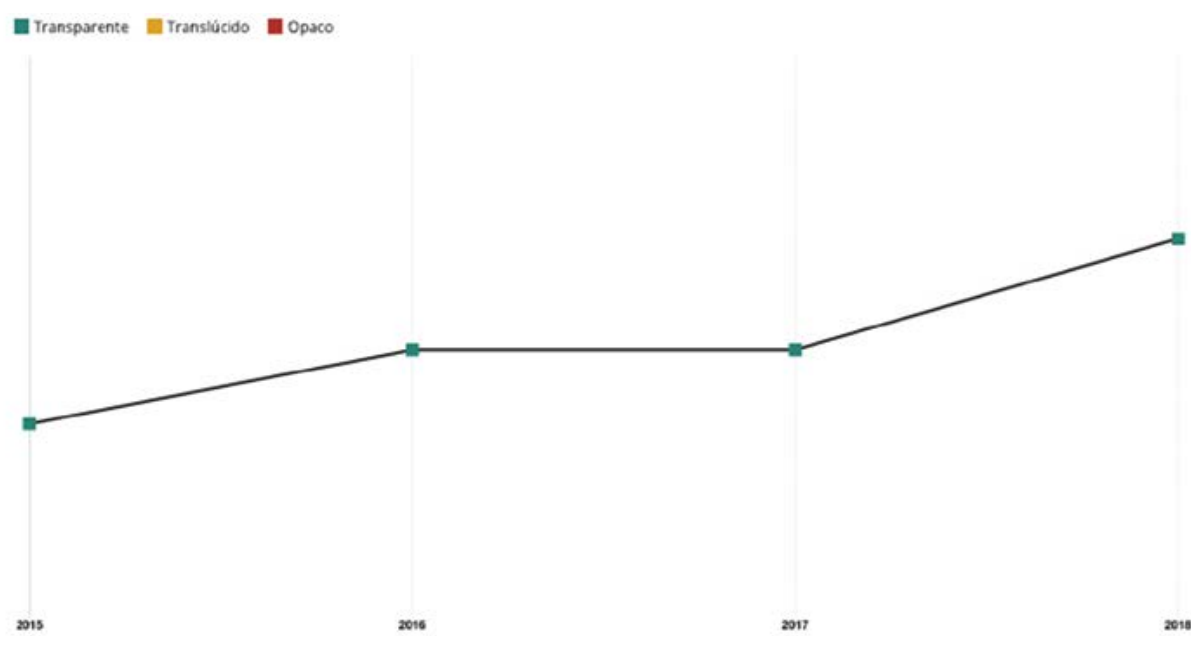

Fuente: Fundación Compromiso y Transparencia.

Otra experiencia destacable es la del Mapa InfoParticipa, que elabora una medición de la transparencia de decenas de ayuntamientos de todo el país y otorga un sello de reconocimiento. A diferencia de TI y Compromiso y Transparencia, InfoParticipa no procede de una ONG o una fundación, sino que es fruto de un proyecto de $\mathrm{i}+\mathrm{D}+\mathrm{I}$ financiado por el Ministerio de Economía y Competitividad. En su página web $^{6}$ indican que su metodología consta de 52 indicadores en forma de pregunta que se valoran de forma positiva solo en el caso de que la información sea hallada en tres clics o menos en la web.

Una organización que ha ganado mucha relevancia mediática en este ámbito, como señala Sierra (2018), es Dyntra (Dynamic Transparency). Es administrada por la organización belga sin ánimo de lucro Dyntra ivzw y se organiza de forma colaborativa, de manera que múltiples usuarios ayudan en la evaluación de la transparencia de muy distintas instituciones, tanto públicas, como privadas. A partir de estas colaboraciones, se elaboran rankings a través de su propia plataforma digital. La metodología es binaria, es decir, solamente se valora si un elemento es publicado o no en la página web. Ello permite elaborar una clasificación según el porcentaje obtenido por las instituciones evaluadas. Dyntra también ofrece una evolución cronológica del cumplimiento de sus indicadores en forma de gráfico. Además,

${ }_{6}$ Disponible en: https://www.mapainfoparticipa.com/index/home/4. 
una cuestión interesante de este ranking es que, junto a cada elemento cumplido por una institución, se ofrece el enlace en el que se ha hallado.

Se ha observado que estos índices de transparencia no basan sus metodologías en las obligaciones de la Ley 19/2013, sino que imponen unos criterios propios. En este sentido, surge una pregunta: ¡cómo se eligen estos indicadores y por qué? Esta información resulta de gran importancia, más aún si se tiene en cuenta que se valoran de la misma manera unos datos y otros. Es decir, el índice de Dyntra suma la misma cantidad de puntos si un ayuntamiento publica la nómina de los concejales que si difunde los datos anuales sobre absentismo laboral.

Además, como ya habían señalado otros autores (Sierra, 2018; Ros Medina, 2018), los rankings de cada organización cuentan con indicadores muy distintos, pero no se conoce su origen. ¿Por qué Transparencia Internacional cuenta con un indicador que es "Canales o sistemas específicos de denuncia para los afiliados o ciudadanos» en su índice de partidos políticos y Dyntra o la Fundación Compromiso y Transparencia no?

Ello conduce a que las instituciones obtengan resultados muy diferentes en los índices de cada organización. Por ejemplo, el partido político Ciudadanos obtuvo una calificación del $100 \%$ en el índice de Transparencia Internacional de 2017 (el último realizado por la organización), mientras que consiguió un 55,5\% en el de Dyntra y un 65,5\% en la Fundación Haz.

Es aquí donde radica uno de los principales problemas de estos índices de transparencia: al contar con metodologías tan dispares, los resultados pueden variar enormemente y ser utilizadas por las instituciones para lanzar un mensaje de higiene democrática. Aunque un ayuntamiento tenga un mal resultado en un índice, es posible que obtenga un resultado mejor en otro, que puede utilizar en su propio beneficio para mejorar su imagen. De hecho, se observa que algunos portales de transparencia cuentan con estructuras basadas en las de los propios indicadores de algunas organizaciones.

Se considera positivo que existan este tipo de índices de transparencia, pues el examen de las instituciones las invita a competir por ser la más transparente y ello deriva en un aumento de las informaciones publicadas en los portales de transparencia. Aun así, sería interesante que los organismos completasen esa fiscalización puramente cuantitativa con otro tipo de actividades de monitorización de la transparencia, que pueden ser de tipología cualitativa. También sería de enorme relevancia conocer la justificación de los indicadores en los índices de transparencia elaborados, puesto que ello puede servir para mejorar los estudios en publicidad activa y seguir desarrollando los portales de las instituciones.

$7 \quad$ Fecha de consulta: 19-01-2021. 


\section{CONCLUSIONES}

España cuenta con una joven y escasa cultura de la transparencia, que puede verse desarrollada si se impulsan iniciativas de control y fiscalización de la apertura informativa. Estas actividades presionan a las instituciones y las obligan a mejorar su comportamiento en el ámbito de la transparencia, con el objetivo de mitigar la desconfianza de la ciudadanía, luchar contra la corrupción y regenerar las instituciones democráticas.

En este artículo, hemos diferenciado entre la monitorización pública de la transparencia y la que proviene de la sociedad civil. La fiscalización pública la ejerce el Consejo de Transparencia y Buen Gobierno a nivel estatal y los comisionados correspondientes en el ámbito territorial. Estos consejos no existen en todas las comunidades, lo cual demuestra un gran desnivel territorial en la aplicación y monitorización de la transparencia en España. Se puede afirmar que el sistema de autonomías refuerza las desigualdades entre comunidades respecto a la evaluación de la transparencia.

Urge que las comunidades autónomas que no cuentan con un órgano público fiscalizador (Asturias, Cantabria, Castilla La Mancha y Extremadura) de la transparencia los constituyan y pongan en marcha, con el objetivo de controlar la aplicación de las normas y garantizar que se cumple el derecho de acceso a la información de la ciudadanía. Esto no solo implicará una evolución de la apertura informativa en dichas autonomías, sino que también entorpecerá menos la labor del CTBG, que tiene suscritos convenios con estas para ejercer esa labor.

El CTBG se encarga de controlar el cumplimiento de la norma a nivel estatal y lo hace recibiendo las reclamaciones de acceso a la información de los ciudadanos, así como analizando la publicidad activa de las instituciones obligadas. Para ello, utiliza la metodología MESTA, que permite cuantificar tanto la cantidad de información publicada como la forma en la que esta se comparte.

Se trata de una metodología muy completa, aunque se han identificado varios problemas al respecto. En primer lugar, es estudio cuantitativo, pero sería interesante también contar con algunos elementos cualitativos que permitieran a las instituciones aprender a gestionar la transparencia de forma más eficiente. En segundo lugar, resulta complicado utilizar MESTA cuando surgen problemas imprevistos, como la actual pandemia. Es indispensable que en momentos así, el órgano público garante de la transparencia analice el trabajo de las instituciones. Por último, el gran problema del CTBG radica en los límites de personal y recursos con los que cuenta. El reglamento dificulta enormemente una mayor inversión en el organismo y ello provoca que el CTBG no pueda ejercer correctamente su labor. De hecho, en su Memoria de 
actividades de 2019 solo analizaron la publicidad activa de una muestra de organizaciones, dadas estas limitaciones.

En este artículo se quiere hacer mención también de un posible peligro de estos consejos. Se debe evitar la politización de estas instituciones evaluadoras públicas, para garantizar la autonomía de la fiscalización de la transparencia, que no debe obedecer a tendencias ideológicas. Por tanto, estos deben poder ser críticos con las administraciones públicas independientemente del partido que gobierne, tanto a nivel estatal como autonómico.

La monitorización cívica de la transparencia, por su parte, puede complementar el trabajo de estos organismos públicos. Se observa la existencia de diferentes monitorizaciones en este sentido: aquellas que provienen de organizaciones sin ánimo de lucro (como ONG y fundaciones) y las correspondientes a investigaciones académicas.

Los organismos cívicos de monitorización de la transparencia suelen recurrir a la realización de índices o rankings basados en el cumplimiento total o parcial de una serie de indicadores. En este sentido, una de los elementos que se cuestionan es la elección de unos indicadores en cada uno de estos rankings. Las diferencias entre los indicadores escogidos por los distintos organismos conllevan unos resultados muy diferentes por parte de las instituciones. Un ayuntamiento podría ser el más transparente en un índice y obtener una posición más mediocre en otro por este motivo, lo que implica que pueden ser resultados contradictorios según el índice del que se trate. Ello puede ser utilizado por las propias instituciones para mejorar su reconocimiento mediático y político.

Se propone también que los organismos impulsen otras actividades monitorizadoras para complementar el trabajo que ya hacen. Estos organismos deben ser independientes de las instituciones a las que evalúen, para actuar con imparcialidad. Algunas propuestas para el desarrollo de nuevas actividades son:

- Análisis cualitativo de la transparencia de las instituciones, que permita llegar a conclusiones reveladoras sobre la forma de trabajar y de defender la transparencia por parte de las instituciones y entidades privadas.

- Control y asesoramiento acerca del derecho de acceso a la información.

- Reutilización de los datos publicados por las instituciones para demostrar su utilidad en la formación de una ciudadanía informada. Se pueden realizar aplicaciones, periodismo de datos o de verificación, entre otros.

Esto se une a la labor de control, que sirve para presionar a los políticos a impulsar la transparencia, reformarla y regenerar las instituciones. Además, 
también tienen como objetivo formación de la ciudadanía y la deben seguir desarrollando para el crecimiento y el fortalecimiento de la cultura de la transparencia en España. La innovación y la accesibilidad a esta monitorización es esencial para defender los valores de la transparencia y que tenga consecuencias reales en la actividad de las instituciones.

Uno de los principales retos para futuras investigaciones consiste crear un censo con los organismos de monitorización de la transparencia en Espańa. Gracias a este, se podrían clasificar según el tipo de actividades fiscalizadoras que ejerzan.

No cabe duda de que defender la transparencia es hoy en día un signo de salud democrática. Para fortalecerla y, por tanto, impulsar la regeneración de las estructuras políticas y la ruptura de las barreras con la ciudadanía, es precisa una evaluación constante de su ejecución. Por ello, la inversión en los órganos fiscalizadores y el desarrollo de sus metodologías son indispensables. Así, esta monitorización de la transparencia, que puede desarrollarse tanto desde los órganos públicos como desde los civiles, podrá medir la adecuación de las políticas de transparencia a las necesidades sociales y tecnológicas actuales.

\section{Bibliografía}

Bennett, W. L. y Livingston, S. (2018). The disinformation order: Disruptive communication and the decline of democratic institutions. European Journal of Communication, 33 (2), 122-139. Disponible en: https://doi.org/10.1177/0267323118760317.

Berliner, D. (2014). The Political Origins of Transparency. The Journal of Politics, 76 (2), 479-491. Disponible en: https://doi.org/10.1017/S0022381613001412.

Bertot, J. C., Jaeger, P. T. y Grimes, J. M. (2010). Using ICTs to create a culture of transparency: E-government and social media as openness and anti-corruption tools for societies. Government Information Quarterly, 27 (3), 264-271. Disponible en: https://doi. org/10.1016/j.giq.2010.03.001.

Cahlikova, T. y Mabillard, V. (2020). Open Data and Transparency: Opportunities and Challenges in the Swiss Context. Public Performance and Management Review, 43 (3), 662-686. Disponible en: https://doi.org/10.1080/15309576.2019.1657914.

Consejo de Transparencia y Buen Gobierno (2020). Memoria de actividades del año 2019. Disponible en: https://bit.ly/3uNqed7.

Curto-Rodríguez, R. (2020). Transparencia operativa de las comunidades autónomas espańolas mediante sus portales de datos abiertos. El Profesional de la Información, 29 (1), 1-14. Disponible en: https://doi.org/10.3145/epi.2020.ene.15.

Delgado Morales, F., López Carvajal, F. J. y Sierra Rodríguez, J. (2015). Regulación y sistemas de evaluación de la transparencia en España. Revista de Derecho de La Hacienda Pública, 5, 111-134. Disponible en: http://revista.cgr.go.cr/.

Feenstra, R. A. (2012). Democracia monitoriza en la era de la nueva galaxia mediática. Barcelona: Icaria. Disponible en: https://doi.org/10.15448/1984-6746.2012.1.11229. 
Hood, C. y Heald, D. A. (2006). Transparency: The key to better governance? Oxford: Oxford University Press. Disponible en: https://doi.org/10.5871/bacad/9780197263839.001.0001.

Janssen, M., Charalabidis, Y. y Zuiderwijk, A. (2012). Benefits, adoption barriers and myths of open data and open government. Information Systems Management, 29 (4), 258-268. Disponible en: https://doi.org/10.1080/10580530.2012.716740.

Keane, J. (2009). The life and Death of Democracy. London: Simon and Schuster.

Mabillard, V. y Zumofen, R. (2017). The complex relationship between transparency and accountability: A synthesis and contribution to existing frameworks. Public Policy and Administration, 32 (2), 110-129. Disponible en: https://doi.org/10.1177/0952076716 653651.

Magallón Rosa, R. (2019). La (no) regulación de la desinformación en la Unión Europea. Una perspectiva comparada. Revista de Derecho Político, 106, 319-347. Disponible en: https://doi.org/10.5944/rdp.106.2019.26159.

Matheus, R., Janssen, M. y Janowski, T. (2020). Design principles for creating digital transparency in government. Government Information Quarterly, 38 (1), 101550. https://doi. org/10.1016/j.giq.2020.101550.

Molina Rodríguez-Navas, P., i Solà, N. S. y Rius, M. C. (2017). Metodologías de evaluación de la transparencia: procedimientos y problemas. Revista Latina de Comunicación Social, 72, 818-831. Disponible en: https://doi.org/10.4185/RLCS-2017-1194.

Pernagallo, G. y Torrisi, B. (2020). A logit model to assess the transparency of Italian public administration websites. Government Information Quarterly, 37 (4), 101519. Disponible en: https://doi.org/10.1016/j.giq.2020.101519.

Ramió, C. (2019). Inteligencia artificial y administración pública: robots y humanos compartiendo el servicio público. Madrid: Los Libros de la Catarata.

Red de Consejos y Comisionados de Transparencia (2020). Declaración de la UNED. Disponible en: https://bit.ly/3my1X6Z.

Ros Medina, J. L. (2018). La evaluación de la transparencia en España a debate metodológico: MESTA e índices de transparencia internacional. Revista Internacional Transparencia e Integridad, 6, 1-22.

Ruijer, E., Détienne, F., Baker, M., Groff, J. y Meijer, A. J. (2020). The Politics of Open Government Data: Understanding Organizational Responses to Pressure for More Transparency. American Review of Public Administration, 50 (3), 260-274. Disponible en: https://doi.org/10.1177/0275074019888065.

Ruvalcaba-Gómez, E. A., Criado, J. I. y Gil-García, J. R. (2018). Discussing open government as a concept: A comparison between the perceptions of public managers and current academic debate. ACM International Conference Proceeding Series. 1-10. Disponible en: https://doi.org/10.1145/3209281.3209320.

Sierra Rodríguez, J. (2018). Mediciones y premios de transparencia. Revista Española de Transparencia, 7, 71-97. Disponible en: https://doi.org/10.51915/ret.71.

Worthy, B. (2010). More open but not more trusted? The effect of the freedom of in- formation act 2000 on the United Kingdom central government. Governance, 23 (4), 561-582. Disponible en: https://doi.org/10.1111/j.1468-0491.2010.01498.x. 
